\section{Ebola: models do more than forecast}

Your assertion that models of the Ebola epidemic have failed to project its course misrepresents their aims (see Nature 515, 18; 2014). They helped to inspire and inform the strong international response that may at last be slowing the epidemic (see M. F. C. Gomes et al. PLoS Curr. Outbreaks http://doi.org/ vvd; 2014).

Subsequent models assessed the likely impact of different public-health interventions and policy decisions (J. A. Lewnard et al. Lancet Infect. Dis. 14, 1189-1195 (2014) and A. Pandey et al. Science http://doi.org/wts; 2014). As those interventions were implemented and as people's behaviour changed, case counts below the modelled baseline were early indicators that the response to the outbreak was having an effect.

Epidemics are affected by countless variables, so uncertainty is a given. Models synthesize available information. Without them, there is little to guide decision-makers during an outbreak. Their importance goes beyond providing forecasts. Caitlin Rivers ${ }^{*}$ Virginia Bioinformatics Institute, Virginia Tech, Blacksburg, USA. cmrivers@vbi.vt.edu ${ }^{*}$ On behalf of 24 correspondents (see go.nature.com/dfzbde for a full list).

\section{Ebola: the power of behaviour change}

Without including social, cultural and behavioural responses to the Ebola epidemic, models may overestimate outbreak size (Nature 515, 18; 2014).

Behavioural response, triggered by an epidemic, can slow down or even stop virus transmission (see S. Funk et al. Proc. Natl Acad. Sci. USA 106, 6872-6877; 2009). Indeed, altered cultural perception in response to the disease enabled people's behaviour to change in ways that helped to contain outbreaks in the past (see B. S. Hewlett and R. P. Amola Emerg. Infect. Dis. 9, 1242-1248; 2003).

Reports from Foya in Liberia indicate that the outbreak there is now in decline. A local information campaign to change funeral practices and other behaviours seems to have paid off.

More aid and more personnel are urgently needed, but so is the involvement of local communities and the provision of information that can help to contain this epidemic.

Sebastian Funk, Gwenan M. Knight London School of Hygiene \& Tropical Medicine, London, UK. Vincent A. A. Jansen Royal Holloway University of London, Egham, Surrey, UK. vincent.jansen@rhul.ac.uk

\section{Can brain training boost cognition?}

Eminent scholars from around the world last month signed a statement on the "brain training' industry (see go.nature. $\mathrm{com} / \mathrm{d} 2 \mathrm{bpuj})$. They point out discrepancies between current scientific understanding of cognitive enhancement and advertising claims for commercial cognitive-training software. But it should not be inferred that software can never improve cognition (see D. Bavelier et al. Nature Rev. Neurosci. 12, 763-768; 2011).

The effectiveness of 'braintraining' software may differ, so product claims might not always be exaggerated. And, given that new training programs are compared with results from control groups engaging in other stimulating activities, the absence of any effect after training can be relative, and is not necessarily definitive. Cognitive enhancement is a vast enterprise that has not yet been clearly defined, and finding optimal ways to train the brain is still a promising area of research. David Moreau Princeton
University, New Jersey, USA. dmoreau@princeton.edu

\section{Chinese universities: beware cronyism}

Jie Zhang rightly points out that China's universities need high-quality faculty members if they are to be competitive internationally (Nature 514, 295-296, 2014). But there are risks in giving individual colleges and departments more autonomy in recruiting staff.

Excessive recruitment has led to bloating and inefficiency in some Chinese universities, which might be exacerbated by greater autonomy. More recruiting freedom could also encourage cronyism, which already undermines research and higher education in China (see, for example, go.nature.com/vl4rxt; in Chinese)

To avoid these pitfalls and to ensure the quality and diversity of new faculty members, university panels should appoint the strongest academic performers from a shortlist drawn up by departmental recruiting panels in an open and transparent process.

Hong-Wei Xiao China Agricultural University, Beijing, China.

xhwcaugxy@163.com

\section{Chinese universities: gear up for Nobels}

We agree with Jie Zhang that university reform is needed to improve the quality of Chinese research papers (Nature 514, 295-296; 2014). A home-grown scientist in China might then stand a chance of winning a Nobel prize for the first time.

Novelty in research remains rare: publications with a Chinese scientist as first or corresponding author accounted for just 362 papers in Nature and 388 in Science from 1992 to 2012. This is despite the government's increased investment in research during 2002-12 and its launching of talent schemes and large scientific programmes in past decades. China needs to recognize that Nobel prizes are awarded for scientific breakthroughs, not for short-term successes.

Switching the country's emphasis from publications in journals in Thomson Reuters' Science Citation Index to multiple evaluation criteria could help, and would offset academic corruption in promotions and student graduation.

China should implement fixed annual salaries for scientists, rather than paying their incomes as a component of research funding, which undermines motivation. The scientific administration system needs overhauling, particularly with respect to funding applications and individual performance evaluation.

Yi-Ping Chen, Yi-Shan Lin, Yi Zhang Institute of Earth Environment, Chinese Academy of Sciences, Xian, China. chenyp@ieecas.cn

\section{Rename comet probe after Greek hero}

The Rosetta spacecraft's Philae probe, which landed successfully on an orbiting comet on 12 November (see Nature http:// doi.org/w8k; 2014), could be renamed Pheidippides - for its record-setting marathon run and transmission of its message before collapsing.

Len Fisher University of Bristol, $U K$.

len.fisher@bristol.ac.uk

\section{CORRECTION}

The Outlook article 'The search for the rice of the future' (Nature 514, S60-S61; 2014) wrongly stated that a flood-resistant gene was bred into rice by Pamela Ronald. In fact, the breeding was done by David Mackill, Abdelbagi Ismail and their team at IRRI. 